\title{
Chicken Nugget lässt Speiseröhre platzen
}

Um sich mit einer Hühnermahlzeit die Speiseröhre zu durchlöchern, ist es offenbar nicht nötig, einen zum Huhn gehörigen Knochen zu verschlucken. Dafür genügt bereits ein Chicken Nugget, wie ein Fall aus Kanada zeigt.

Chicken Nuggets erfreuen sich vor allem bei jüngeren Essern einiger Beliebtheit. Ein wesentlicher Bestandteil der panierten und frittierten Klumpen aus Hühnermasse ist Formfleisch: getumbeltes, also mechanisch gewalktes Fleisch. Der Verbund der Fasern soll sich lockern und Eiweiß austreten. Beigemischt werden Bindemittel, Trockenmilch, Essig und Cornflakesbrösel, um nur einige Zutaten zu nennen. Manchem mag dies mehr nach Labor als nach Küche klingen. Tatsächlich war Robert Baker, der die Nuggets vor über 50 Jahren erfunden hat, Lebensmittelwissenschaftler.

Das Klebefleisch der Nuggets sollte dem Kau- und Schluckapparat eigentlich keine allzu großen Probleme bereiten. Ein 25-jähriger Kanadier musste jedoch am eigenen Leib erfahren, dass das nicht immer der Fall sein muss. Blass, kurzatmig und mit epigastrischen Schmerzen wurde der junge Mann in der Notaufnahme des Toronto General Hospital vorstellig. Anamnestisch war zu erfahren, dass er eine Stunde zuvor ein Chicken Nugget geschluckt hatte. Dabei habe er einen scharfen, kratzenden Schmerz empfunden. Erbrochen habe er nicht.

\section{Aufschlussreiches Thoraxröntgen}

Das Abdomen war vor allem epigastrisch schmerzempfindlich und zeigte deutliche Abwehrspannung. Die Laborwerte waren weitgehend unauffällig, von leichten Erhöhungen der Leukozyten, des Bilirubins und der Amylase abgesehen.

Die Computertomografie ergab eine Ruptur des distalen Ösophagus mit Kontrastmittelaustritt, ein Pneumomediastinum und einen Plauraerguss links. Nach der Verordnung von Nahrungskarenz, Flüssigkeit i.v. und Breitspektrumantibiotika verlegten die Mediziner den Mann auf die Thoraxchirurgie. Ösophagoskopisch ließ sich die Diagnose einer nichtiatrogenen Ösophagusruptur und eines Boerhaave-Syndroms bestätigen. Der Patient bekam einen Ösophagusstent, um die Perforation abzudecken.

Nach fünf Tagen trat Fieber mit Leukozytose und zunehmenden Brustschmerzen auf. Das CT zeigte nun einen Mediastinalabszess und einen rechtsseitigen Erguss. Den Abszess drainierten die Thorxchirurgen, zugleich dekortizierten sie den rechtsseitigen Pleuraraum. Drei Wochen später konnte der Stent entfernt werden, die Ruptur des Ösophagus war verheilt.

\section{Boerhaave-Syndrom: hohe Letalität}

Für die Notfallmediziner um Jo Jo Leung (Toronto), die über diese Geschichte berichteten, birgt der Fall zwei wesentliche Lehren. „Erstens: Nichtiatrogene, lebensbe- drohliche Ösophagusrupturen können sich ereignen, ohne dass Erbrechen, Anfälle oder chronischer Husten in der Anamnese auftauchen“. Auch ohne einschlägige Anamnese könne sich ein Boerhaave-Syndrom entwickeln, dessen Letalität zwischen $20 \%$ und $75 \%$ liege. Der Verdacht müsse sich regen, wenn Patienten kurzatmig seien und über epigastrische Schmerzen klagten. „Und zweitens“, so Leung weiter, „kann offenbar auch knochen- und schalenfreie weiche Nahrung den intraösophagealen Druck dermaßen erhöhen, dass es für eine Ruptur ausreicht.“

Vom Nugget-Erfinder Robert Baker wird kolportiert, er habe zunächst wenig darauf gegeben, ob Menschen seine Kreationen bekömmlich fanden. Nur wenn der Hund sie nicht fraß, sei er zur Überarbeitung nochmals zurück in sein Labor gegangen.

(Robert Bublak)

Aga Z et al. Case Rep Emerg Med 2016 (online first)

\section{H. pylori - und sonst noch?}

\section{Risikofaktoren des Magenkarzinoms}

H. pylori ist und bleibt der wichtigste Einflussfaktor auf das Magenkarzinomrisiko. Doch wie eignen sich der Kaffeekonsum oder diagnostizierte Gastritiden, um das Risiko abzuschätzen?

Die Assoziation von Kaffee- und Teekonsum und der Magenkarzinominzidenz wurde in einer Untersuchung mit 477.312 Teilnehmern betrachtet. Im Follow up von 11,6 Jahren konnte kein Einfluss auf die Häufigkeit von Adenokarzinomen erkannt werden. Jedoch zeigte sich ein Zusammenhang zwischen Kaffee und dem Risiko für
Kardiakarzinome. In Schweden wurden Daten von 405.172 Patienten mit präkanzerösen Veränderungen untersucht. Bei allen wurde zwischen 1979 und 2011 eine Magenbiopsie durchgeführt und Karzinome, die frühestens zwei Jahre nach der Endoskopie auftraten, erfasst. Es ergab sich ein Risiko von 1:256 bei normaler Mukosa, 1:85 bei Gastritis, 1:50 bei atropher Gastritis, 1:39 bei intestinaler Metaplasie und 1:19 bei Dysplasie.

(Kathrin von Kieseritzky)

basierend auf: Vortrag von Prof. Wolfgang Fischbach, Aschaffenburg, Februar 2016 